\title{
Short-term results following two-stage revision for periprosthetic joint infection
}

\author{
$1 \mathrm{MBChB}(\mathrm{UCT})$; Registrar \\ $2 \mathrm{MBChB}(\mathrm{UP})$, FCOrth(SA); Consultant \\ 3 MBChB, FCOrth(SA), MMed(Orth)(Wits); Consultant \\ $4 \mathrm{MBBCh}($ Wits); Registrar \\ 5 MD, FCOrth(SA); Professor and Head of Arthroplasty
}

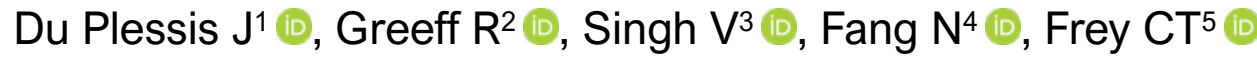

Department of Orthopaedic Surgery, Chris Hani Baragwanath Academic Hospital, University of the Witwatersrand, Johannesburg, South Africa

Corresponding author: Dr Jason du Plessis, PO Box 2696, Noordheuwel, Krugersdorp, 1756; tel: 082445 7247; email: Jasondp_29@yahoo.com

\begin{abstract}
Background: Hip and knee arthroplasty procedures are successful surgical procedures, with total hip arthroplasty being named the operation of the 20th century. With there being an estimated rate globally of periprosthetic joint infection of $1 \%$ for hips and $2 \%$ for knees, this minimal infection rate represents a large global concern. The successful management of periprosthetic joint infection remains controversial with multiple proposed strategies. Our aim is to present our short-term data for a two-stage revision protocol.

Methods: A single centre retrospective review of an existing database starting from January 2013 and including April 2019 was conducted looking at patients having undergone two-stage revision for periprosthetic joint infection. The unit utilised a standard approach to two-stage revisions. Data was collected from the existing database to ascertain short-term success based on the Delphibased international multidisciplinary consensus criteria.

Results: A total of 2125 entries were reviewed from the database comprising 1912 primary arthroplasty procedures. From all revision cases 19 patients were identified to have undergone a two-stage revision by our unit. Of these patients we managed to collect sufficient data to gauge treatment success in 12 patients. Of these 12 patients with a mean follow-up of 25.6 months, ten reported complete wound healing, pain improvement and no subsequent surgery. One patient demised from septic complications and one required subsequent arthrodesis which controlled the sepsis.

Conclusion: Our results showed a high infection eradication rate following our two-staged revision protocol despite frequent delays between first and second stages as a result of resource constraints and limitations.
\end{abstract}

Level of evidence: Level 4

Keywords: revision, arthroplasty, two-stage, periprosthetic joint infection

Citation: Du Plessis J, Greeff R, Singh V, Fang N, Frey CT. Short-term results following two-stage revision for periprosthetic joint infection. SA Orthop J 2020;19(2):64-69. http://dx.doi.org/10.17159/2309-8309/2020/v19n2a1

Editor: Dr Thomas Hilton, University of Cape Town, South Africa

Received: August 2019

Accepted: October 2019

Published: May 2020

Copyright: $\odot 2020$ Du Plessis J. This is an open-access article distributed under the terms of the Creative Commons Attribution Licence, which permits unrestricted use, distribution and reproduction in any medium, provided the original author and source are credited.

Funding: No funding was received for this study.

Conflict of interest: None of the authors have any conflicts of interest to declare. 


\section{Introduction}

Hip and knee arthroplasty procedures are considered highly successful surgical procedures, with total hip arthroplasty being named the operation of the 20th century. ${ }^{1}$ Globally, there is an increase in the number of these procedures due to an increasing elderly population; an increase in skilled surgeons; and the success rate of the operation. ${ }^{2}$ With an estimated rate globally of periprosthetic infection of $1 \%$ for hips and $2 \%$ for knees, ${ }^{3}$ even a minimal infection rate represents a large global concern medically ${ }^{2}$ and financially. ${ }^{4}$

The successful management of periprosthetic joint infection (PJI) remains controversial, with multiple successful treatment options reported in the literature leading to uncertainty as to which is the best approach. The two-staged approach is still regarded as the gold standard 5 for chronic infections defined as an infection after six to eight weeks from the index procedure.6,7 The two-staged approach has reported cure rates of over $90 \%, 8,9$ ranging from $76 \%$ to $100 \%$. This figure depends on the definition of cure which has not been uniform throughout the literature ${ }^{5}$ with some authors only looking at re-operation and others looking at loosening or combinations thereof. ${ }^{10}$ Within the body of literature looking at the two-stage approach to PJI, there is controversy regarding the specific aspects of each stage ${ }^{5}$ in terms of type of cement spacer, antibiotic dose within the spacer, time between stages and the relevance of further investigations such as an alpha-defensin test at the second stage.

The purpose of this study was to assess the short-term success of our two-stage revision protocol in patients presenting with late chronic periprosthetic joint infection as defined by Segawa et al. ${ }^{11}$ and further expanded on by Tsukayama ${ }^{12}$ and summarised in Table / following hip and knee arthroplasty at our institution.

\section{Patients and methods}

Institutional approval was obtained and a retrospective review conducted of patients managed for chronic deep PJI using a twostage revision protocol. Information was extracted from a patient database and included patients managed at our institution from January 2013 up to and including April 2019. The database, which was recorded on Apple Numbers (Apple Inc, Cupertino, CA USA), was analysed and all patients with radiographic evidence of having a cement spacer were included in the initial cohort. All patients with cement spacers were regarded to have been treated for a late chronic PJI as defined by Tsukayama, ${ }^{11,12}$ depicted in Table I. Thereafter patients who had been assessed following reimplantation and had documented outcomes were included. Patients with no record of follow-up following reimplantation were excluded from the cohort.

Demographic data and details of respective surgical procedures were collected onto a spreadsheet using Microsoft Excel (Microsoft, Redmond, WA, USA). We documented the joint involved, the number of surgeries and the dates these were performed as well as the outcome of the most recent available assessment of the patient recorded.

PJI was routinely diagnosed based on the Musculoskeletal Infection Society (MSIS) criteria for PJI of $2011^{13}$ prior to the initiation of a two-stage revision. After diagnosis patients were booked for and underwent a two-staged revision. The first stage consisted of a thorough joint debridement of all infected and necrotic tissue as well as removal of all implants. Biopsies were sent from a minimum of five sites within the debrided joint, including from bone and synovium, for histological and microbiological analysis. Cement spacers were made using casting moulds provided by Zimmer Biomet (Zimmer Biomet, Warsaw IN, USA). The cement spacers were made using $80 \mathrm{~g}$ Palacos $\mathrm{R}+\mathrm{G}$ (Haraeus, Hanau, Germany) containing $1 \mathrm{~g}$ gentamycin with $3 \mathrm{~g}$ vancomycin and $3 \mathrm{~g}$ cefuroxime added. The cement was then allowed to harden in the moulds and implanted into the dead space as an articulating spacer.

Post-operatively patients were placed on six weeks of antibiotics, with intravenous vancomycin and oral rifampicin being the firstline empiric agents. After culture and sensitivity were obtained, antibiotics were tailored to the cultured organism and where possible oral agents were given (cloxacillin or linezolid with rifampicin) and the patients were discharged to continue their sixweek course as outpatients. Following the six weeks of antibiotics, a minimum period of two weeks was allowed before repeat blood investigations were done, including white cell count, C-reactive protein, erythrocyte sedimentation rate and ferritin-iron ratio. If these were suppressed after the antibiotic-free period, the second stage was planned.

The second stage consisted of removal of the cement spacers and a repeat debridement of the joint and bone ends. In cases where the eradication was clinically questionable, an alphadefensin test was planned to be done; if positive, a repeat first stage would be performed, and where negative, we would proceed with the second stage. Multiple biopsies were again taken at the time of reimplantation, and reimplantation with revision implants was performed based on the bone defects encountered for each case, an example of which can be seen in Figure 1. This was done despite evidence indicating that reimplantation cultures often did not correlate with poorer outcomes. ${ }^{5,14}$ Following the second stage operation, patients were treated for another six weeks with antibiotics based on the original first stage culture results and tailored if needed based on the repeat culture results.

The success of this systematic intervention was based on the Delphi-based international multidisciplinary consensus criteria for the resolution on PJI published in 2013. ${ }^{10}$ Here, success was defined as: 1) no clinical failure (healed wound without fistula or draining sinus and pain-free joint, and no recurrence by the same organism; 2) no subsequent surgical intervention after reimplantation for infection; 3) no death caused by a condition linked to PJI.

\section{Results}

We reviewed a total of 2125 entries for all arthroplasty procedures performed between January 2013 and April 2019, with 1912 entries for primary arthroplasty procedures done. Of the primary arthroplasty procedures done, total hip arthroplasty accounted for 1041 entries and total knee arthroplasty accounted for 871 entries. From these entries, 19 patients were identified to have undergone two-stage revisions for chronic PJI, with eight hips and 11 knees in that cohort. There were no patients documented to have required

Table I: Classification of PJI based on clinical presentation ${ }^{12}$

\begin{tabular}{|l|l|l|l|l|}
\hline Timing & Type 1 & Type 2 & Type 3 & Type 4 \\
\hline Dositive intra-operative & $\begin{array}{l}\text { Early post-operative } \\
\text { infection (A: superficial, } \\
\text { B: deep) }\end{array}$ & $\begin{array}{l}\text { Acute haematogenous } \\
\text { infection }\end{array}$ & Late chronic infection \\
\hline Definition & $\begin{array}{l}>2 \text { positive intra-operative } \\
\text { cultures }\end{array}$ & $\begin{array}{l}\text { Infection within } 30 \text { days of } \\
\text { surgery }\end{array}$ & $\begin{array}{l}\text { Haematogenous seeding in } \\
\text { well-functioning prosthesis }\end{array}$ & $\begin{array}{l}\text { Chronic infection present } \\
>30 \text { days }\end{array}$ \\
\hline
\end{tabular}



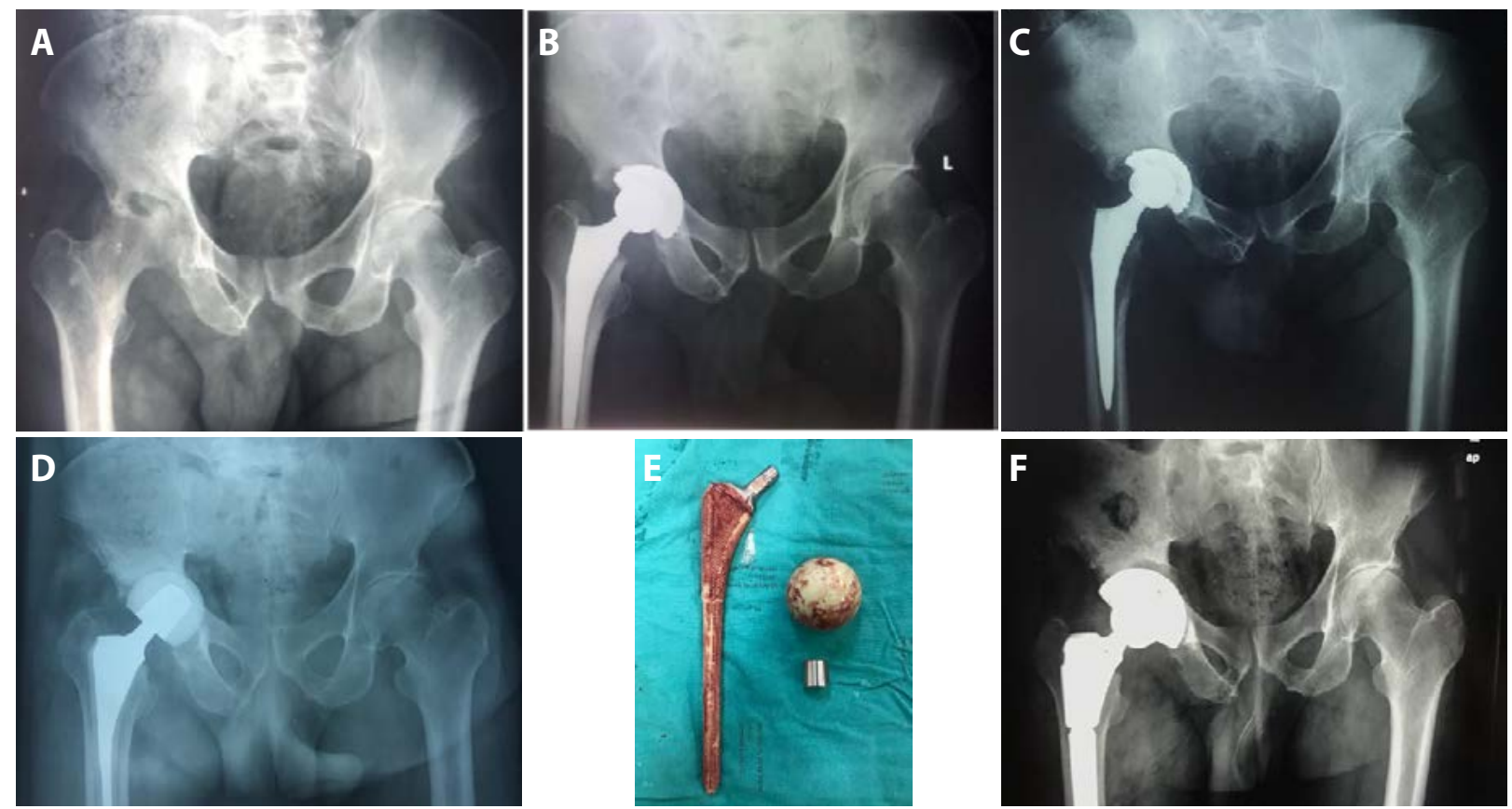

Figure 1. Case representing a two-stage hip revision. A: initial pre-operative radiograph showing right hip avascular necrosis; B: post primary total hip arthroplasty; C: proximal femur and acetabular lucency when presenting with draining sinus; D: first stage debridement with articulating cement spacer, with spacer removed at second stage (E); F: final implants following second stage reimplantation

a repeat first stage for questionable clearance and reimplantation. This correlates to an overall chronic infection rate of $0.99 \%(0.76 \%$ for hips and $1.26 \%$ for knees) as defined by Tsukayama. ${ }^{12}$ With regard to these findings, it should be kept in mind that our institution is a referral centre and some revisions were done for primary joints done at other hospitals. Similarly, we operate on patients from a large demographic area and some complications may not have returned to our institution but rather been managed elsewhere.

Of note there were also a total of 79 patients identified who were managed for acute post-operative periprosthetic joint infections (Tsukayama type 2). Of these, 51 were hips and 28 were knees. This correlated to an acute infection rate of $4.13 \%$ (4.9\% for hips, 3.2\% for knees). These patients were managed with debridement and implant retention (DAIR) with exchange of modular components, followed by six weeks of targeted antibiotics based on intraoperative culture specimens. There were no patients noted to have presented with acute haematogenous infection (Tsukayama type 3). Of the patients identified with acute infection, none progressed to two-stage revision following initial surgical management.

From the identified 19 patients with Tsukayama type 4 infection, 12 were found to have documented follow-up data which allowed them to be assessed according to the aforementioned Delphi-based international multidisciplinary consensus definition of success (five hips, seven knees). The remaining patients had insufficient data on record or had not followed up again at our institution for assessment and were thus excluded from the study. The outcomes relating to treatment success were captured and tabulated (Table II). All patients undergoing revision were classified as type B hosts according to McPherson et al. ${ }^{15,16}$ who modified the CiernyMader classification for osteomyelitis (Table III) to include specific local and systemic factors (Table IV).

Table II: Patient details and outcomes

\begin{tabular}{|c|c|c|c|c|c|c|}
\hline Patient & Joint involved & $\begin{array}{l}\text { Time between revision } \\
\text { stages (months) }\end{array}$ & $\begin{array}{c}\text { Time since } \\
\text { reimplantation (months) }\end{array}$ & Wound healed & Pain & $\begin{array}{l}\text { Subsequent } \\
\text { surgeries }\end{array}$ \\
\hline 1 & Hip & 24 & 36 & Yes, no sinus & Improved & No \\
\hline 2 & Hip & 5 & 4 & Yes, no sinus & Improved & No \\
\hline 3 & Knee & 5 & 6 & Yes, no sinus & Improved & No \\
\hline 4 & Knee & 4 & 6 & Yes, no sinus & Improved & No \\
\hline 5 & Knee & 29 & 29 & Yes, no sinus & None & No \\
\hline 6 & Hip & 3 & 34 & Yes, no sinus & None & No \\
\hline 7 & Knee & 10 & 17 & Yes, no sinus & Improved & No \\
\hline 8 & Hip & 6 & 24 & Yes, no sinus & Improved & No \\
\hline 9 & Knee & 6 & 28 & Yes, no sinus & None & No \\
\hline 10 & Hip & RIP & RIP & RIP & RIP & RIP \\
\hline 11 & Knee & 5 & 20 & Yes, no sinus & Improved & No \\
\hline 12 & Knee & 7 & 78 & Yes, no sinus & None & Knee fusion \\
\hline
\end{tabular}


Table III: Host classification by McPherson ${ }^{16}$

\begin{tabular}{|l|l|}
\hline Type A & \multicolumn{1}{c|}{ Uncompromised, normal host } \\
\hline Type B & $\begin{array}{l}\text { Compromised host with one or two local and/or } \\
\text { systemic factors }\end{array}$ \\
\hline Type C & $\begin{array}{l}\text { Significant compromise with more than two } \\
\text { compromising factors or one of the following } \\
\\
\text { - Neutrophils }<1000 \\
\text { - CD4 count }<100 \\
\text { - Intravenous drug user } \\
\text { - Other chronic active infection } \\
\text { - Haematological malignancy }\end{array}$ \\
\hline
\end{tabular}

Table IV: Local and systemic compromising factors as described by McPherson ${ }^{16}$

\begin{tabular}{|c|c|}
\hline Systemic factors & Local factors \\
\hline $\begin{array}{l}\text { Age }>80 \text { years } \\
\text { Diabetes } \\
\text { Immunosuppressive medication } \\
\text { Systemic inflammatory disease } \\
\text { (RA, SLE) } \\
\text { Malignancy } \\
\text { Hepatic insufficiency (cirrhosis) } \\
\text { Chronic indwelling catheter } \\
\text { Renal failure requiring dialysis } \\
\text { Pulmonary insufficiency } \\
\text { Alcoholism } \\
\text { Chronic malnutrition } \\
\text { Current nicotine use } \\
\text { Systemic immune compromise }\end{array}$ & $\begin{array}{l}\text { Active infection }>3-4 \text { months } \\
\text { Synovial cutaneous fistula } \\
\text { Soft tissue loss from prior trauma } \\
\text { Vascular insufficiency (arterial or } \\
\text { venous) } \\
\text { Prior peri-articular fracture } \\
\text { (crush) } \\
\text { Multiple previous incisions (skin } \\
\text { bridge) } \\
\text { Prior local irradiation to wound } \\
\text { area } \\
\text { Subcutaneous abscess }>8 \mathrm{~cm}^{3}\end{array}$ \\
\hline
\end{tabular}

From the 12 patients with completed data there were four males and eight females, with a mean age at follow-up of 61.66 years (range 51-75, median 61). The mean follow-up time for the cohort was 25.6 months (range 4-78, median 24), with the mean time between the first and second stages being 9.45 months (range $3-29$, median 6). One patient was found to have demised following the first stage of the revision process as a result of severe sepsis secondary to systemic Pseudomonas aeruginosa. A second patient had recurrence of sepsis two years after the second stage, and subsequently underwent a knee fusion. All remaining patients $(10 / 12,83.3 \%)$ had complete wound healing with no erythema or recurrence of sinus tract formation. Four patients reported no residual joint pain, with seven reporting some residual pain but an improvement compared to before the revisions.

\section{Discussion}

The diagnosis and management of $\mathrm{PJI}$ remains controversial with no definitive diagnostic tests ${ }^{17}$ or clear universally accepted management protocol or definition of treatment success. The diagnosis is based on a combination of clinical examination findings and laboratory results. ${ }^{17}$ The Musculoskeletal Infection Society (MSIS) together with the Infectious Disease Society of America (IDSA) previously developed criteria aimed at standardising the definition and diagnosis of PJI in 2011. ${ }^{13}$ These criteria were the recently revised into a scoring system ${ }^{17}$ to add weighting to different aspects of the criteria and possibly improve diagnostic accuracy.

The management of PJI is as contentious as the diagnosis, with multiple proposed treatment algorithms used in the literature. The literature becomes more confusing as there have been multiple definitions of treatment success or treatment failure, thus making the comparison of reported success rates difficult (Table V). ${ }^{5}$ Because of these diverse criteria for success, we used the Delphibased international multidisciplinary consensus definition. ${ }^{10}$ Twostage revision remains the gold standard for chronic PJI, ${ }^{2}$ but success has also been reported for one-stage revision performed under strict conditions. ${ }^{18,19}$

Within the realms of two-stage revision there remains controversy regarding the preferred protocol.5,20,21 Our unit followed a similar protocol as described by Sukeik et al. ${ }^{2}$ as far as possible, with some alteration to these steps based on the constraints of an overburdened public health system with long waiting lists and occasional drug availability constraints. This led to our patients having an extended spacer retention time averaging 9.45 months (range 3-29 months, median 6 months). Fu et al. ${ }^{22}$ found the optimal timing of reimplantation to be between 12 and 16 weeks, but had a mean spacer retention time of 24 weeks in their success group ${ }^{22}$ whereas our time to reimplantation translates to approximately 110 weeks. Despite these challenges our short-term results remain promising with $83.3 \%$ of reviewed patients showing good wound healing and improvement in pain and function without subsequent surgery.

Other authors have proposed some variation to the described two-stage approach. Chung et al. recently described a protocol of two-stage debridement but with retention of implants. ${ }^{23}$ They

Table V: Reported success rates following two-stage revision for PJI (adapted from Akgün et al. ${ }^{5}$ )

\begin{tabular}{|c|c|c|c|}
\hline $\begin{array}{c}\text { Study } \\
\text { (individually referenced in above } \\
\text { article by Akgün et al.) }\end{array}$ & $\begin{array}{l}\text { Number of } \\
\text { patients }\end{array}$ & Definition of failure & Rate of infection eradication (\%) \\
\hline Chen et al. & 155 hips & $\begin{array}{l}\text { Repeated operation } \\
\text { Long-term antibiotics }\end{array}$ & 91.7 \\
\hline Oussedik et al. & 39 hips & Recurrent infection & 96 \\
\hline Tan et al. & $\begin{array}{l}186 \text { knees } \\
81 \text { hips }\end{array}$ & Delphi-based definition & 76 \\
\hline Lange et al. & 82 hips & Kamme et al. & 85.4 \\
\hline Triantafyllopoulos et al. & $\begin{array}{l}239 \text { knees } \\
261 \text { hips }\end{array}$ & $\begin{array}{l}\text { Wound healing problems } \\
\text { Elevated ESR/CRP } \\
\text { Long-term suppression }\end{array}$ & 91.2 \\
\hline Fink et al. & 36 hips & $\begin{array}{l}\text { Clinical signs of infection } \\
\text { CRP more than } 10 \mathrm{mg} / \mathrm{dl} \\
\text { Osteolysis }\end{array}$ & 100 \\
\hline Berend et al. & 186 hips & Further surgery for infection & 83 \\
\hline Ibrahim et al. & 125 hips & Recurrence of infection & \\
\hline Leung et al. & 50 hips & Recurrence of infection & 79 \\
\hline
\end{tabular}


reported an overall success rate of $86.7 \%$ (93.8\% in primary and $77.1 \%$ in revision arthroplasty) after an average follow-up of 41.8 months (12-171). In their protocol the modular components were removed, scrubbed and sterilised in the autoclave or antimicrobial soak then reinserted with antibiotic-impregnated cement beads. These beads were removed at repeat debridement five days later. ${ }^{23}$

Other aspects of controversy affecting our methodology involve the relevance of a positive alpha-defensin test at the reimplantation stage. Our protocol was to repeat the first stage procedure in the presence of a positive result. This practice is costly and time consuming for the unit and the patient. Samuel et al. ${ }^{24}$ found that at one year a positive alpha-defensin at reimplantation correlated poorly with the presence of persistent infection and recommended against its routine use in patients with cement spacers. ${ }^{24}$ This finding is postulated to be a result of a possible inflammatory reaction to the cement spacer leading to higher false positive results. ${ }^{25}$ Frozen section has been shown to correlate well with infection being present at time of reimplantation, ${ }^{22}$ but this was not part of our protocol as the service has been unavailable in the past, with staffing and resource constraints being cited as barriers to the service availability.

Complication rates for two-stage revisions are varied, with Ibrahim et al..$^{26}$ noting a high mortality rate $(15 \%)$ in their study cohort, although there was no comparison group, whereas our cohort had a single sepsis-related mortality documented (8.3\%). Higher rates of subsequent dislocation have also been reported, ${ }^{27}$ with a $9 \%$ dislocation rate compared to the quoted $0.5 \%-5.3 \%$ dislocation rate in primary arthroplasty depending on the approach used. ${ }^{28}$ Our cohort reported no such complications in the patients we were able to review. The possibility remains, however, that some unreviewed patients may have demised or dislocated since reimplantation but this was not ascertained.

Limitations of our study are the small numbers of patients undergoing revision reviewed and the short follow-up of the cohort relating to the recent nature of the digital database. The electronic database was also not completed for all patients, resulting in the exclusion of seven patients which, in a small cohort, could prove to be significant.

\section{Conclusion}

A high short-term infection cure rate was achieved using a standardised two-stage revision protocol despite institution-related alterations regarding time to reimplantation. Further follow-up is required to ascertain the medium- and long-term success of this management protocol, and the time to reimplantation should be shortened to be more in line with larger published studies.

\section{Ethics statement}

Prior to commencement of the study, ethical approval was obtained from the following ethical review board: HREC (Wits) clearance certificate M170415 (obtained $29 / 05 / 2017$, annual update 27/02/2019). The authors declare that this submission is in accordance with the principles laid down by the Responsible Research Publication Position Statements as developed at the 2 nd World Conference on Research Integrity in Singapore, 2010.

All procedures were in accordance with the ethical standards of the responsible committee on human experimentation (institutional and national) and with the Helsink Declaration of 1975, as revised in 2008.

\section{Declaration}

The authors declare authorship of this article and that they have followed sound scientific research practice. This research is original and does not transgress plagiarism policies.

\section{Author contributions}

JdP contributed to the conception and design of the work; the acquisition, analysis, and interpretation of data for the work; drafting the work; final approval of the version to be submitted to the journal and ongoing input regarding corrections and editing. RG conceptualised the work and contributed to design and interpretation of the work. Further contributions include critical revision and expression of intellectual content, as well as final approval of the version submitted to the journal.

VS contributed to design of the work, analysis and interpretation of the data as well as revision and final approval.

$\mathrm{NF}$ contributed to the collection of the data as well as approval of the final version. CF conceptualised the work and contributed to design and interpretation of the work. Further contributions include critical revision and expression of intellectual content, as well as final approval of the version submitted to the journal.

\section{ORCID}

Du Plessis J (D) https://orcid.org/0000-0002-4617-9742

Greeff R iD https://orcid.org/0000-0001-7691-5192

Singh V (iD) https://orcid.org/0000-0002-7876-2493

Fang N (iD https://orcid.org/0000-0002-9020-1556

Frey CT (iD https://orcid.org/0000-0003-1692-9749

\section{References}

1. Learmonth ID, Young C, Rorabeck C. The operation of the century: total hip replacement. Lancet. 2007;370(9597):1508-19.

2. Sukeik M, Haddad FS. Periprosthetic joint infections after total hip replacement: an algorithmic approach. SICOT-J. 2019;5

3. Kapadia BH, Berg RA, Daley JA, et al. Periprosthetic joint infection. Lancet. 2016;387(10016):386-94.

4. Vanhegan I, Malik A, Jayakumar P, UI Islam S, Haddad F. A financial analysis of revision hip arthroplasty: the economic burden in relation to the national tariff. $J$ Bone Joint Surg Br. 2012;94(5):619-23.

5. Akgün D, Müller M, Perka $C$, Winkler T. High cure rate of periprosthetic hip joint infection with multidisciplinary team approach using standardized two-stage exchange. J Orthop Surg Res. 2019;14(1):78.

6. Karim MA, Andrawis J, Bengoa F, et al. Hip and knee section, diagnosis, algorithm: Proceedings of international consensus on orthopedic infections. J Arthroplasty. 2019;34(2):S339-S50.

7. Sukeik M, Patel S, Haddad FS. Aggressive early debridement for treatment of acutely infected cemented total hip arthroplasty. Clin Orthop Relat Res. 2012;470(11):3164-70.

8. Sukeik M, Haddad FS. Two-stage procedure in the treatment of late chronic hip infections-spacer implantation. Int J Res Med Sci. 2009;6(5):253.

9. Berend KR, Lombardi AV, Morris MJ, et al. Two-stage treatment of hip periprosthetic joint infection is associated with a high rate of infection control but high mortality. Clin Orthop Relat Res. 2013;471(2):510-18

10. Diaz-Ledezma C, Higuera CA, Parvizi J. Success after treatment of periprosthetic joint infection: a Delphi-based international multidisciplinary consensus. Clin Orthop Relat Res. 2013;47(7):2374-82

11. Segawa H, Tsukayama DT, Kyle RF, Becker DA, Gustilo RB. Infection after total knee arthroplasty: a retrospective study of the treatment of eighty-one infections. JBJS. 1999;81(10):1434-45.

12. Tsukayama DT, Goldberg VM, Kyle R. Diagnosis and management of infection after total knee arthroplasty. JBJS. 2003;85:75-80.

13. Parvizi J, Zmistowski B, Berbari EF, et al. New definition for periprosthetic joint infection: from the Workgroup of the Musculoskeletal Infection Society. Clin Orthop Relat Res. 2011;469(11):2992.

14. Bejon $P$, Berendt $A$, Atkins $B$, et al. Two-stage revision for prosthetic joint infection: predictors of outcome and the role of reimplantation microbiology. J Antimicrob Chemother. 2010;65(3):569-75.

15. Cierny G MJ, Penninick JJ. A clinical staging system for adult osteomyelitis. Contemp Orthop. 1985;10:17-37.

16. McPherson EJ, Woodson $\mathrm{C}$, Holtom $\mathrm{P}$, et al. Periprosthetic total hip infection: outcomes using a staging system. Clin Orthop Relat Res (1976-2007). 2002;403:8-15.

17. Parvizi J, Tan TL, Goswami K, et al. The 2018 definition of periprosthetic hip and knee infection: an evidence-based and validated criteria. J Arthroplasty. 2018;33(5):1309-14. e2.

18. Zahar A, Klaber I, Gerken A-M, et al. Ten-year results following one-stage septic hip exchange in the management of periprosthetic joint infection. J Arthroplasty. 2019;34(6):1121-26. 
19. Zeller V, Lhotellier L, Marmor S, et al. One-stage exchange arthroplasty for chronic periprosthetic hip infection: results of a large prospective cohort study. JBJS. 2014;96(1):e1.

20. Tan TL, Gomez MM, Manrique J, Parvizi J, Chen AF. Positive culture during reimplantation increases the risk of subsequent failure in two-stage exchange arthroplasty. JBJS. 2016;98(15):1313-19.

21. Puhto A-P, Puhto TM, Niinimäki TT, Leppilahti JI, Syrjälä HP Two-stage revision for prosthetic joint infection: outcome and role of reimplantation microbiology in 107 cases. J Arthroplasty. 2014;29(6):1101-104.

22. Fu J, Ni M, Li H, et al. The proper timing of second-stage revision in treating periprosthetic knee infection: reliable indicators and risk factors. J Orthop Surg Res. 2018;13(1):214.

23. Chung AS, Niesen MC, Graber T, et al. Two-stage debridement with prosthesis retention for acute periprosthetic joint infections. $J$ Arthroplasty. 2019;34(6):1207-13.

24. Samuel LT, Sultan AA, Kheir M, et al. Positive alpha-defensin at reimplantation of a two-stage revision arthroplasty is not associated with infection at 1 year. Clin Orthop Relat Res. 2019;477(7):1615-21.

25. Singh G, Deutloff N, Maertens N, et al. Articulating polymethylmethacrylate (PMMA) spacers may have an immunomodulating effect on synovial tissue. Bone Joint J. 2016;98(8):1062-68.

26. Ibrahim M, Raja S, Khan M, Haddad F. A multidisciplinary team approach to two-stage revision for the infected hip replacement: a minimum five-year follow-up study. Bone Joint $J$. 2014;96(10):1312-8.

27. McAlister IP Perry KI, Mara KC, et al. Two-stage revision of total hip arthroplasty for infection is associated with a high rate of dislocation. JBJS. 2019;101(4):322-29.

28. Moretti VM, Post ZD. Surgical approaches for total hip arthroplasty. Indian J Orthop. 2017;51(4):368. 